\title{
INTERPRETIVE SUMMARIES, JULY 2011
}

Invited review: Associations between variables of routine herd data and dairy cattle welfare indicators. By de Vries et al., page 3213. A promising strategy to monitor animal welfare more efficiently is to first estimate the level of animal welfare on a farm, based on routine herd data that are available in national databases. Our aim was to identify routine herd data that are associated with dairy cattle welfare indicators in a literature review. We found that many variables of routine herd data were associated with welfare indicators, especially those relating to milk yield, culling, and reproduction. We concluded that routine herd data have potential to estimate the level of animal welfare on dairy farms.

Diversity of lactic acid bacteria associated with traditional fermented dairy products in Mongolia. By Yu et al., page 3229. The diversity of lactic acid bacteria isolated from natural fermented dairy products in Mongolia were accurately described in this study, which will provide some raw data and strain resource for further studies of probiotic strain selection and starter culture design concerning the industrial production of traditional fermented milk.

$\beta$-Galactosidase activity of commercial lactase samples in raw and pasteurized milk at refrigerated temperatures. By Horner et al., page 3242. Lactose-free milk is manufactured using enzymes called lactases. These enzymes break down the milk sugar lactose into simple sugars that are easily digested. Lactases generally work best at temperatures above room temperature. This study explored the effectiveness of 4 commercially available lactase enzymes when used at refrigerated temperatures at different concentrations in different types of milk. The findings show that it is feasible to use these lactases at refrigerated temperatures, thus allowing for a flexible manufacturing process for lactose-free milk, which may reduce its cost.

Determination of molecular weight of a purified fraction of colloidal calcium phosphate derived from the casein micelles of bovine milk. By Choi et al., page 3250. The colloidal calcium phosphate (CCP) that is present in casein micelles plays a critical role in the formation, stability, and physical properties of caseins. The CCP is present as small (2 to $3 \mathrm{~nm}$ ) granules or nanoclusters. We attempted to estimate the molecular weight of CCP by enzymatic digestion of caseins to create a CCP-casein phosphopeptide fraction and we estimated the molecular weight of this fraction using size-exclusion chromatography coupled with multi-angle laser light scattering. Our estimated size for CCP was comparable to our theoretical prediction.
Lactococcus strains treated with heat and henegg-white lysozyme induce abundant interleukin-12 production by J774.1 macrophages and murine spleen cells. By Aoki et al., page 3262. Lactococcus strains treated with heat and hen-egg-white lysozyme induced abundant interleukin-12 production. They were more hydrophobic than heat-killed bacteria and were engulfed by macrophages easily. Moreover, they had stronger resistance to lysozyme and macrophage digestion. Lysozyme-modified Lactococcus strains produced by this method could be potent immunomodulators for activating cellular immunity. These findings have important implications for the design of dairy products that have the immunomodulatory effect using the modified bacteria.

Effect of probiotic yogurt containing Lactobacillus acidophilus and Bifidobacterium lactis on lipid profile in individuals with type 2 diabetes mellitus. By Ejtahed et al., page 3288. Dyslipidemia is an important risk factor for cardiovascular disease in type 2 diabetes mellitus. In this study, we investigated the effects of probiotic and conventional yogurt on blood lipids in individuals with type 2 diabetes. Subjects consumed daily $300 \mathrm{~g}$ of probiotic yogurt containing Lactobacillus acidophilus La5 and Bifidobacterium lactis $\mathrm{Bb} 12$ or $300 \mathrm{~g}$ of conventional yogurt for $6 \mathrm{wk}$. Serum total cholesterol and low-density lipoprotein cholesterol concentrations were significantly decreased in the probiotic group compared with the control group. Therefore, Lactobacillus acidophilus La5 and Bifidobacterium lactis Bb12 may contribute to improvement of the lipid profile.

Effect of polymorphisms in the leptin, leptin receptor, and acyl-CoA:diacylglycerol acyltransferase 1 (DGAT1) genes and genetic polymorphism of milk proteins on cheese characteristics. By Glantz et al., page 3295. Cheese production has increased worldwide during the last decade and is expected to increase within the coming decade. By including genetic markers for cheese quality within breeding programs, the economic output of the breeding and dairy industries would most likely increase. This study indicated that the leptin, leptin receptor, $\beta$-lactoglobulin, and $\kappa$-casein genes have an effect on technological properties important for cheese production and hence, have the potential to act as genetic markers for cheese characteristics within genetic selection programs.

Short communication: Profile of virulence factors of Staphylococcus aureus isolated from subclinical bovine mastitis in the state of Rio de 
Janeiro, Brazil. By Coelho et al., page 3305. Staphylococcus aureus produces proteins that contribute to ability to colonize the mammary gland and enhance the capacity to cause bovine mastitis. This study characterized some virulence factors in Staph. aureus isolates from milk samples of subclinical mastitis from dairy cattle farms located in Rio de Janeiro, Brazil. These findings are valuable to understanding the distribution of Staph. aureus strains in Rio de Janeiro.

Incidence of metabolic disorders and reproductive performance following a short (35-d) or conventional (60-d) dry period management in commercial Holstein herds. By Santschi et al., page 3322. A period of rest of $60 \mathrm{~d}$ is general practice for dairy cows between consecutive lactations, but recent studies suggest that a dry period of 30 to $35 \mathrm{~d}$ could be more beneficial for today's high-producing dairy cows. Few studies have looked at the effects of shorter dry periods on health and metabolism, although these traits are of great economic importance for producers. Results of the present experiment were that a short dry period could reduce the incidence of ketosis with no major negative effects on health and reproduction and could therefore be beneficial for today's cows.

Bovine neuronal vesicular glutamate transporter activity is inhibited by ergovaline and other ergopeptines. By Xue et al., page 3331. Cattle grazing endophyte-infected tall fescue have reduced productive and reproductive capacities. We found that ergopeptines contained in endophyte-infected tall fescue can inhibit the function of a glutamate transport activity that is specific for glutamate signaling in nervous and peripheral tissues. Thus, a causative mechanism for the widespread physiologic effects of fescue toxicosis was identified.

Concentration of progesterone during the development of the ovulatory follicle: I. Ovarian and embryonic responses. By Cerri et al., page 3342. Greater concentrations of progesterone before and after insemination are associated with improved fertility. Progesterone alters ovarian and uterine function and could directly or indirectly influence fertilization and early stages of embryo development. Reduced concentrations of progesterone during the ovulatory follicle development had a major effect on concentrations of estradiol during proestrus and follicular dynamics, but resulted in similar oocyte fertilization and only minor changes in embryo quality. Effects of progesterone during follicle development might occur either later on embryo development or on the uterine environment.

Concentration of progesterone during the development of the ovulatory follicle: II. Ovarian and uterine responses. By Cerri et al., page 3352.
Increased concentrations of progesterone before and after insemination are associated with improved fertility. Progesterone is important for ovarian follicle development and to prepare the uterus to receive and maintain the conceptus. Reduced concentrations of progesterone during follicular development increased the basal concentrations of luteinizing hormone, leading to a larger ovulatory follicle with more estradiol and less insulinlike growth factor-1 in the follicular fluid. Furthermore, reduced concentrations preceding ovulation resulted in earlier upregulation of endometrial estrogen receptor- $\alpha$, increased concentration of prostaglandin $\mathrm{F}_{2 \alpha}$ metabolite, and increased occurrence of short luteal phases, which might explain some of the reduction in fertility in cows with low progesterone.

Efficacy of a novel internal dry period teat sealant containing $0.5 \%$ chlorhexidine against experimental challenge with Streptococcus uberis in dairy cattle. By Petrovski et al., page 3366. The objective was to estimate the incidence of clinical mastitis and infection status at calving after experimental challenge with Streptococcus uberis in quarters treated with a novel internal dry period teat sealant that contains chlorhexidine compared with commercial teat sealant and untreated control cows. Clinical mastitis during the early dry period occurred in 1.2, 1.8, and $26.8 \%$ and intramammary infections due to challenge and other organisms at calving in 15,32 , and $79 \%$ of quarters treated with a novel teat sealant, a commercial teat sealant, and untreated controls respectively.

Using water to cool cattle: Behavioral and physiological changes associated with voluntary use of cow showers. By Legrand et al., page 3376. Water is commonly used to cool cattle in summer either at milking or over the feed bunk, but little research has examined how dairy cows voluntarily use water separate from these locations. When given the opportunity, cattle spent on average $3 \mathrm{~h}$ and up to $8 \mathrm{~h} / 24 \mathrm{~h}$ in a cow shower. Use of the shower reduced body temperature relative to that of control cows. The variability between cows indicates that the behavioral response to water is an important consideration in the design of sprinkler systems used for summer cooling.

Diagnosing intramammary infections: Evaluation of composite milk samples to detect intramammary infections. By Reyher and Dohoo, page 338\%. Composite milk samples are used for detection of intramammary infection (IMI) as they are economical, easier to collect than individual quarter samples, and may be useful when considering management options at the cow and herd level. Because the causative organism for an IMI is initially unknown, the reliability of composite samples for detection of all types of mastitiscausing bacteria is important. Composite samples 
showed moderate sensitivity and high specificity for a number of pathogens. Predictive values from composite sampling can be useful in field scenarios to assist mastitis management.

Risk factors associated with short-term posttreatment outcomes of clinical mastitis. By Pinzón-Sánchez and Ruegg, page 339\%. This study characterized outcomes of cases of mild and moderate clinical mastitis that occurred in dairy cows located on commercial dairy farms in Wisconsin. The results of this study can directly benefit dairy producers when making strategic treatment decisions by characterizing post-treatment outcomes and demonstrating the association of risk factors with successful outcomes posttreatment.

Detection of multiple Mycoplasma species in bulk tank milk samples using real-time PCR and conventional culture and comparison of test sensitivities. By Justice-Allen et al., page 3411. Mycoplasmas cause several important disease syndromes in dairy cattle. In this study, the sensitivity of a real-time PCR assay was compared to conventional culture for the detection of mycoplasmas in bulk tank milk samples. In addition to demonstrating comparable sensitivity, the PCR detected and facilitated the identification of multiple mycoplasma species in a single sample. The presence of Mycobacterium bovis, M. alkalescens, M. arginini, M. bovigenitalium, and M. gateae was confirmed by analysis of the PCR-generated amplicon (melting temperature, length, and DNA sequence). This realtime PCR protocol has utility in routine diagnostic testing of milk samples.

Ranking experts' preferences regarding measures and methods of assessment of welfare in dairy herds using Adaptive Conjoint Analysis. By Lievaart and Noordhuizen, page 3420. Different concepts have been described for the assessment of animal welfare in dairy herds, but not much consensus exists among welfare experts on the traits addressed in these concepts, nor on the methods used to quantify these traits. The Adaptive Conjoint Analysis technique was used to rank the preferences of 24 internationally acknowledged European welfare experts towards 70 possible traits and various methods to assess welfare measures in dairy herds. The measures "lameness cases" and "competition for feed and water" received the highest scores with a relatively small range between the scores of the experts.

Differential regulation of bovine pyruvate carboxylase promoters by fatty acids and peroxisome proliferator-activated receptor- $\alpha$ agonist. By White et al., page 3428. Glucose and carbon metabolism are critical during transition to lactation in dairy cows. Pyruvate carboxylase (PC) activity is critical to the rate of gluconeogenesis from lactate and carbon flux into the tricarboxylic acid cycle. Three unique promoter regions have been identified for bovine $\mathrm{PC}$ and while regulation of $\mathrm{PC}$ promoters has been observed in other species, regulatory control of bovine PC by promoters has not yet been examined in bovine. Stearic acid and peroxisome proliferator-activated receptor- $\alpha$ agonist have a role in regulation of bovine PC through promoter regulation. Understanding the regulation of this key enzyme could provide valuable insight into future management of transition cows.

Efficacy of embryo transfer in lactating dairy cows during summer using fresh or vitrified embryos produced in vitro with sex-sorted semen. By Stewart et al., page 343\%. Transfer of fresh embryos produced in vitro using sex-sorted semen into lactating dairy recipients during the summer resulted in greater pregnancy and calving rates compared to either transfer of vitrified embryos derived in vitro or artificial insemination. Fresh embryos produced in vitro with sex-sorted semen can be used effectively during the summer to improve fertility in lactating dairy cows and increase the percentage of heifer calves born. Further improvements are needed to make the transfer of vitrified, in vitro produced embryos an effective tool to mitigate the effects of heat stress on fertility in lactating dairy cattle.

Lactose in blood plasma and the ability of dairy cows to tolerate once-daily milking in terms of milk loss and milk recovery. By Guinard-Flament et al., page 3446. Once-daily milking reduces dairy-farm workload over a short or long period and is potentially useful, provided that cows tolerate this practice by showing low decreases in milk yield, low residual effects on milk when returned to twice-daily milking, and no negative effects on udder health. This study addressed the issue of the ability of dairy cows to tolerate oncedaily milking by examining lactose in blood plasma as an indicator of mammary epithelium integrity.

Cefquinome sulfate behavior after intramammary administration in healthy and infected cows. By Zonca et al., page 3455. The pharmacokinetic profile of cefquinome (CFQ) after repeated intramammary administration in healthy and subclinically infected Staphylococcus aureus cows and the minimum inhibitory concentration (MIC) of Staph. aureus field strains were carried out together with the systemic drug absorption in healthy and infected animals. The systemic drug absorption was low and comparable in healthy and infected animals. The $\mathrm{MIC}_{90}$ value for $\mathrm{CFQ}$ in Staph. aureus field strains $(\mathrm{n}=20)$ was $0.24 \mu \mathrm{g} /$ $\mathrm{mL}$. No differences were observed between infected and healthy quarters. 
Vitamin $D_{2}$ impairs utilization of vitamin $D_{3}$ in high-yielding dairy cows in a cross-over supplementation regimen. By Hymøller and Jensen, page 3462. Vitamin D exists in 2 forms important in the vitamin $\mathrm{D}$ status and supply in cattle: vitamin $\mathrm{D}_{2}$ and $\mathrm{D}_{3}$, which are generally considered of equal physiological value. In the present study, oral administration of a vitamin $\mathrm{D}_{3}$ bolus increased plasma levels of vitamin $\mathrm{D}_{3}$ metabolites more efficiently than oral administration of a vitamin $\mathrm{D}_{2}$ bolus increased plasma levels of vitamin $\mathrm{D}_{2}$ metabolites. Furthermore, a vitamin $\mathrm{D}_{3}$ bolus given 2 wk after a vitamin $\mathrm{D}_{2}$ bolus was less efficient for increasing plasma concentrations of vitamin $\mathrm{D}_{3}$ metabolites than vitamin $\mathrm{D}_{3}$ given without previous vitamin $\mathrm{D}_{2}$ administration.

Transcript abundance of amino acid transporters, $\beta$-casein, and $\alpha$-lactalbumin in mammary tissue of prepartum, lactating, and postweaned sows. By Manjarin et al., page 3467. Modern swine production systems require highly productive animals. Thus, continued development of nutritional strategies to improve milk production and litter weight gain is critical. In this study, genes encoding for 5 amino acid transporters and 2 mammary-synthesized milk proteins were profiled in sow mammary tissue across different stages of mammary physiological activity. A high correlation was found between transcript abundance for 3 of the 5 amino acid transporter genes and that of genes encoding for milk proteins. Therefore, a select number of amino acid transporter genes may represent molecular targets for improvement of sow milk production during lactation.

Effects of sex-sorting and sperm dosage on conception rates of Holstein heifers: Is comparable fertility of sex-sorted and conventional semen plausible? By DeJarnette et al., page 347\%. Holstein heifers were inseminated with $2.1 \times 10^{6}$ or $10 \times 10^{6}$ dosages of sex-sorted or conventionally processed sperm from 8 Holstein sires. Conception rates of both sexsorted and conventional semen were improved by higher sperm dosages. However, conception rates obtained with the $10 \times 10^{6}$ sex-sorted sperm dosage were less than those obtained with either dosage of conventional sperm, implying comparable conception rates for sexsorted and conventional semen may not be plausible in the absence of major advances in sorting technology.

Endocrine changes and liver mRNA abundance of somatotropic axis and insulin system constituents during negative energy balance at different stages of lactation in dairy cows. By Gross et al., page 3484. Endocrine adaptations and effects on hepatic gene expression related to the somatotropic axis were determined during negative energy balance (NEB) immediately postpartum and induced by feed restric- tion during mid lactation. The extent of the changes in endocrine and liver parameters was smaller during a feed restriction-induced NEB than during the NEB in early lactation. The different adaptive reactions of the cow liver to an energy deficiency at the 2 stages in lactation indicate different levels of metabolic priority during the course of lactation.

Short communication: Effect of vitamins E and $\mathrm{C}$ on cortisol production by bovine adrenocortical cells in vitro. By Montalvo et al., page 3495. Supplementation or injection of vitamin E and other antioxidants may reduce stress responses in animals, but the mechanisms are not known. Understanding the mechanism involved in the modulation of stress responses by antioxidants might lead to new strategies to reduce stress in farm animals. A bovine adrenal-cell culture was used to test whether vitamins $\mathrm{E}$ and $\mathrm{C}$ could reduce cortisol production. The modulation effect of vitamins $\mathrm{E}$ and $\mathrm{C}$ on stress response does not take place at the cortex of the adrenal gland.

Technical note: The use of a physical activity monitor to estimate the eating time of cows in pasture. By Ueda et al., page 3498. A commercial physical activity monitor on the neck was used to develop a method for easily estimating the eating time of dairy cows in a pasture. The recording of high activity level ( $\gg 1)$ was recorded associated with the movement of head caused by biting. It was possible to distinguish eating activity from other types of activity with the lowest rate of misclassification of $5.5 \%$. This method of measurement would be a valuable tool in research on the nutritional management of grazing cows.

Technical note: Validation of an ELISA for measurement of tumor necrosis factor alpha in bovine plasma. By Farney et al., page 3504. Measurement of tumor necrosis factor alpha, a key component of inflammatory cascades, has been limited in cattle by a lack of commercially available reagents. A bovinespecific assay was recently introduced, but has not been validated for use in bovine plasma. Our work demonstrates that the assay is capable of precise and accurate quantification of bovine tumor necrosis factor using a modified procedure for plasma samples.

An evaluation of the effect of altering nutrition and nutritional strategies in early lactation on reproductive performance and estrous behavior of high-yielding Holstein-Friesian dairy cows. By Gilmore et al., page 3510. Fertility in the modern highyielding dairy cow has declined markedly over the last $40 \mathrm{yr}$, with only $30 \%$ of animals currently becoming pregnant to first insemination. None of 3 nutritional interventions applied in the current study had a statistically significant effect on reproductive performance of 
Holstein-Friesian cows, but high-starch or low-protein diets in the early postpartum period tended to increase the proportion of animals cycling by d 30 postpartum. In addition, managing the energy balance of cows individually in the early postpartum period increased the $100-d$ in-calf rate.

Effect of varying total mixed ration particle size on rumen digesta and fecal particle size and digestibility in lactating dairy cows. By Maulfair et al., page 352\%. A study was conducted to evaluate the effects of varying total mixed ration particle size on rumen digesta and fecal particle size and digestibility in lactating dairy cows. This study was done to determine the critical size for particles leaving the rumen and whether rumen digesta and fecal particle size change throughout the day. Fecal particle size was not different among rations and averaged $1.13 \mathrm{~mm}$, with over $36 \%$ of particles being larger than $1.18 \mathrm{~mm}$. These results show that the critical size for increased resistance to rumen escape is larger than previously thought, and this critical size is constant throughout the day.

Effect of biotin on milk performance of dairy cattle: A meta-analysis. By Chen et al., page 353\%. A meta-analysis of the effect of biotin on production outcomes of dairy cattle was conducted following a literature review. A fixed effects model was used for analyses. Inclusion of biotin in the diet of lactating cows resulted in increased milk yield and dry matter intake, but had no effect on milk protein and fat concentration.

Hay intake improves performance and rumen development of calves fed higher quantities of milk. By Khan et al., page 354\%. This study compared performance and rumen development of calves fed high volumes of milk $(8 \mathrm{~L} / \mathrm{d}$ from d 3 to $35,4 \mathrm{~L} / \mathrm{d}$ from $\mathrm{d}$ 36 to 53 , and $2 \mathrm{~L} / \mathrm{d}$ for the next $3 \mathrm{~d}$ before weaning at d 56) with and without access to hay. All calves were provided ad libitum access to water and starter. Provision of chopped hay to calves fed high volumes of milk at an early age improved solid feed intake, did not affect body weight gain, and promoted development of reticulorumen.

Effect of intensified feeding of heifer calves on growth, pubertal age, calving age, milk yield, and economics. By Davis Rincker et al., page 3554. Both short- and long-term effects of intensified feeding of heifer calves during the preweaning period were studied. Increasing energy and protein intake from 2 to $42 \mathrm{~d}$ of age resulted in taller, wider, and heavier calves, and tended to reduce age at puberty and calving. Energycorrected milk that was unadjusted for age was not different, but when genetic variation was removed, milk yield tended to be higher in those animals fed the high diet. Intensified feeding of calves allows producers to reduce age at first calving without a detrimental effect on milk yield or economics.

The use of a radiotelemetric ruminal bolus to detect body temperature changes in lactating dairy cattle. By AlZahal et al., page 3568. This study investigated the efficacy of a radiotelemetric rumen temperature sensor in detecting body temperature changes. This technology has the potential to detect such changes in cattle. The capability to monitor body temperature in cattle can improve disease detection, and thus improve animals' health, performance, and welfare.

Residue of melamine and cyanuric acid in milk and tissues of dairy cows fed different doses of melamine. By Sun et al., page 3575. When cows consume melamine tainted feed, either melamine or cyanuric acid (formed from melamine by rumen microorganisms) residues may be found in milk and tissues. This study assessed melamine and cyanuric acid in milk and tissues of dairy cows being dosed with different amounts of melamine. A linear increase in melamine in milk was observed as the dietary intake of melamine increased. Although melamine and cyanuric acid were found to be deposited in some tissues, no cyanuric acid was detected in bovine milk.

Genetic strain and diet effects on grazing behavior, pasture intake, and milk production. By Sheahan et al., page 3583. This study investigated the effects of feeding supplements to dairy cows of North American and New Zealand ancestry on dry matter intake (DMI), milk production, and grazing behavior. North American cows produced more milk and had a greater pasture DMI, although they spent less time grazing. Time spent grazing declined with increased amounts of concentrate DMI. Grazing behavior following morning supplementation was different than that following afternoon supplementation, indicating that different factors were controlling grazing behavior. Understanding how a cow adjusts her grazing behavior in response to feed supplementation is important for the development of strategies that optimize dairy cow production.

Substitution rate and milk yield response to corn silage supplementation of late-lactation dairy cows grazing low-mass pastures at 2 daily allowances in autumn. By Pérez-Prieto et al., page 3592. Feed costs in dairy production systems may be reduced by extending the grazing season into the autumn. During this period, pasture availability is usually low due to low pasture growth rate, the grazing of low-mass pastures being highly probable. The aim of this experiment was to provide guidelines for improving grazing management by evaluating the effect of pasture 
allowance and corn silage supplementation on pasture intake, milk production, and grazing behavior of dairy cows grazing low-mass pastures during autumn.

Short communication: Effects of replacing part of corn silage and alfalfa hay with Leymus chinensis hay on milk production and composition. By Yan et al., page 3605. The objective of this study was to investigate effects of partially replacing corn silage and alfalfa hay with Leymus chinensis hay on milk production and composition. The addition of Leymus chinensis hay increased milk yield, fat content, fat yield, protein yield, and lactose yield. Adding Leymus chinensis hay also decreased somatic cell count in milk. High-quality Leymus chinensis hay could be used as a good forage source for high milk yield and good milk quality in diets of dairy cows.

Short communication: Serum immunoglobulin $\mathrm{G}$ and total protein concentrations in dairy calves fed a colostrum replacement product. By Fidler et al., page 3609. Administration of high-quality colostrum to calves soon after birth is important for maximizing calf health and future productivity. When high-quality colostrum is not available, colostrum replacement products are an alternative. The objective of this study was to evaluate the efficacy of a commercially available plasma-derived colostrum replacement product in dairy calves. One package of this colostrum replacement product did not provide sufficient serum immunoglobulin $\mathrm{G}$ concentration in calves to prevent failure of transfer of passive immunity.

Technical note: Improved methodology for analyses of acid detergent fiber and acid detergent lignin. By Raffrenato and Van Amburgh et al., page 3613. For nutritionists, the lignin method provides useful chemical information to help them determine the maturity and potential digestibility of plant material. The lignin method most often used by ruminant nutritionists relied on asbestos as a filtering aid, but that was removed by the Environmental Protection Agency in 1989. We provide the basis for an updated lignin method with a new glass microfiber filter that meets the criteria of the assay and improves the method.

Evidence that leptin genotype is associated with fertility, growth, and milk production in Holstein cows. By Clempson et al., page 3618. This study investigated single nucleotide polymorphisms (SNP) in the leptin, leptin receptor, and neuropeptide Y (NPY) gene and their associations with growth, milk production, and fertility traits in dairy cows. Leptin, leptin receptor, and NPY SNP were associated with production and fertility traits, whereas leptin SNP were also associated with juvenile growth traits. The associations of leptin SNP with fertility were present in both young heifers and mature cows independent of milk production effects. Leptin and NPY SNP may be useful markers to select dairy cows for optimal growth, fertility and productivity.

Genetic parameters for milk somatic cell score and relationship with production and udder type traits in dairy Alpine and Saanen primiparous goats. By Rupp et al., page 3629. Mastitis is a major health problem in dairy ruminants. In recent decades, selection programs have been implemented worldwide in cattle and sheep to improve genetic resistance to the disease using mainly milk somatic cell score (SCS) as the selection criterion. This paper reports genetic parameters for the weighted mean of somatic cell scores adjusted for lactation stage in Alpine and Saanen primiparous goats. A reduction in SCS can be achieved by selection in goats with minor consequences on present genetic gains in milk production and udder type traits.

Genetic variation of toll-like receptor genes and infection by Mycobacterium avium ssp. paratuberculosis in Holstein-Friesian cattle. By Ruiz-Larrañaga et al., page 3635. The aim of this study is to provide further clarification of the previously reported associations between toll-like receptor (TLR) genes and susceptibility to infection by Mycobacterium avium ssp. paratuberculosis (MAP) in cattle. Twenty-four single nucleotide polymorphisms (SNP) from TLR1, TLR2 and TLR4 genes have been selected for a population-based genetic association study of a Spanish Holstein-Friesian sample. A risk haplotype in TLR2 gene and 3 SNP in TLR4 have been detected for the first time associated with susceptibility to this infection. Furthermore, among those 3 SNP in TLR4 gene, SNP c.-226G $>$ C localized in the $5^{\prime}$ untranslated region affects the expression of the gene, and this fact could influence individual's response to MAP infection.

Accuracy of genomic prediction using lowdensity marker panels. By Zhang et al., page 3642. Genomic selection has been widely implemented in national genetic evaluations for candidate bulls in the dairy cattle industry, due to its potential advantages over traditional selection methods and the availability of commercial high-density single nucleotide polymorphism (SNP) panels. However, it may not be cost effective for cow selection and for other livestock species. One possible solution is to conduct genomic selection with low-density (LD) SNP panels. In this study, the performances of LD marker panels with evenly-spaced or selected markers were evaluated via simulation with a wide range of scenarios. This study is useful for the design of breeding schemes in the framework of genomic selection with LD panels. 
The use of mid-infrared spectrometry to predict body energy status of Holstein cows. By McParland et al., page 3651. Cow body energy status is an important indicator of health and fertility and can be estimated indirectly through body condition score, but is difficult to measure accurately due primarily to the cost of measuring feed intake. Mid-infrared spectrometry (MIR) is undertaken on all milk samples collected routinely as part of large-scale milk recording and milk payment schemes. This study found that routinely available MIR spectral data is useful in predicting the body energy status of Holstein cows with up to $75 \%$ accuracy throughout lactation. The approach described in this study offers a viable method of routinely predicting energy status on large numbers of commercial cows.

The effect of improving cow productivity, fertility, and longevity on the global warming potential of dairy systems. By Bell et al., page 3662. The global warming potential in carbon dioxide equivalent emissions ( $\mathrm{CO}_{2}$-eq.) and land required for feed of a range of Holstein-Friesian dairy genetic lines $x$ feeding systems were compared and mitigation options suggested. Of the production systems studied, a nongrazing low forage system produced the lowest $\mathrm{CO}_{2}$-eq. emissions and required the least amount of land for feed per unit product, whereas high forage fed cows were limited by their feed intake. Selecting dairy cows on a low or high forage feeding system for efficient feed use offers significant potential for reducing system nutrient requirements, $\mathrm{CO}_{2}$-eq. emissions and land use per unit product.

Effect of imputing markers from a low-density chip on the reliability of genomic breeding values in Holstein populations. By Dassonneville et al., page 3679. A low-density 3,000-marker panel is a cheap alternative to the current 50,000-marker panel. By applying appropriate statistical methods, unknown markers can be imputed using a reference group with some uncertainty. This study investigated the accuracy of imputation from a 3,000-marker to a 50,000-marker panel, and the reliability of genomic prediction using the imputed data. The results show a low imputation error rate and a satisfactory reliability of genomic prediction. Therefore, a 3,000-marker chip imputed to a 50,000-marker panel could be a feasible alternative when pre-screening selection candidates.

Short communication: Influence of the muscle hypertrophy mutation of the myostatin gene on milk production traits and on milk fatty acid composition in dual-purpose Belgian Blue dairy cattle. By Buske et al., page 368\%. The objective of this study was to test if the muscle hypertrophy mutation of the myostatin gene has an influence on milk production traits and on fatty acid composition in cow's milk. This is of importance, because besides the conventional milk production traits (milk, protein, and fat yield), the proportion of the different fatty acids in cow's milk is also important due to human health aspects and technical properties of milk. The results confirmed that the myostatin gene is clearly involved in the conventional milk production traits. Concerning fatty acid composition, a trend for higher saturated fatty acid content in milk was observed for the wild-type allele.

Short communication: Estimates of genetic parameters of body condition score in the first 3 lactations using a random regression animal model. By Loker et al., page 3693. Long-term selection for increased milk production has led to selection for dairy cows with reduced health and fertility. Body condition score (BCS) is an indicator of the energy balance, and may be useful for improving health and fertility. This study estimated genetic parameters of BCS in the first 3 lactations in Canadian Holstein dairy cattle. Much of the variation observed in BCS was controlled by the same genes for each lactation. Regular collection of first-lactation BCS records should be sufficient for genetic evaluation of this trait.

Factors affecting the incidence of first-quality wheels of Trentingrana cheese. By Bittante et al., page 3700. The percentage of wheels evaluated as first quality at 9 and 18 months of ripening was monitored on 386 batches of Trentingrana hard cheese produced by 10 cooperative dairy factories from 2002 to 2008 . Dairy factory explained most of the variability of quality evaluation, followed by season and year of production. The percentage of first-quality wheels decreased between 2002 and 2005, and recovered in the following years. Considerable differences among dairy factories and seasons of production were found. Results support the importance of assessing and monitoring the quality of Trentingrana cheese.

Fortification of Cheddar cheese with vitamin D does not alter cheese flavor perception. By Ganesan et al., page 3708. This article outlines the effect of adding vitamin D to cheese on cheese flavor attributes. Cheese flavor results from a large number of compounds and the inclusion of a new chemical component may alter flavor perception. Our study confirms that vitamin $\mathrm{D}$ can be incorporated into cheese without an appreciable change in cheese flavor perception.

Assessing economic and demographic factors that influence US dairy demand. By Davis et al., page 3715. The dairy industry in the United States offers a wide array of fluid milk and dairy products to consumers. The significant role played by low-fat dairy products in promoting healthy diets, is often emphasized, as is the importance of many other dairy 
products as sources of vitamins and minerals that support bone health. Using Nielsen 2007 data and a censored demand system, 16 products were analyzed. Results indicate that dairy purchases are influenced by price, dairy expenditure, and demographic factors with the latter having marginal, but statistically significant effect on dairy consumption. 